\title{
In vitro metabolism of beclomethasone dipropionate, budesonide, ciclesonide, and fluticasone propionate in human lung precision-cut tissue slices
}

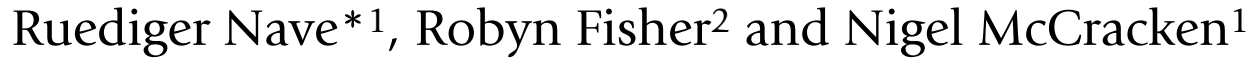

Address: ${ }^{1} \mathrm{DMPK}$, Nycomed GmbH, Konstanz, Germany and ${ }^{2}$ Vitron, Inc., Tucson, Arizona, USA

Email: Ruediger Nave* - ruediger.nave@nycomed.com; Robyn Fisher - robyn@vitron.com; Nigel McCracken - nigel.mccracken@nycomed.com

${ }^{*}$ Corresponding author

Published: 20 September 2007

Respiratory Research 2007, 8:65 doi:10.1186/1465-9921-8-65

This article is available from: http://respiratory-research.com/content/8/1/65

(C) 2007 Nave et al; licensee BioMed Central Ltd.

This is an Open Access article distributed under the terms of the Creative Commons Attribution License (http://creativecommons.org/licenses/by/2.0), which permits unrestricted use, distribution, and reproduction in any medium, provided the original work is properly cited.

\begin{abstract}
Background: The therapeutic effect of inhaled corticosteroids (ICS) may be affected by the metabolism of the drug in the target organ. We investigated the in vitro metabolism of beclomethasone dipropionate (BDP), budesonide (BUD), ciclesonide $(\mathrm{ClC})$, and fluticasone propionate (FP) in human lung precision-cut tissue slices. CIC, a new generation ICS, is hydrolyzed by esterases in the upper and lower airways to its pharmacologically active metabolite desisobutyryl-ciclesonide (des-CIC).
\end{abstract}

Methods: Lung tissue slices were incubated with BDP, BUD, CIC, and FP (initial target concentration of $25 \mu \mathrm{M}$ ) for 2, 6, and $24 \mathrm{~h}$. Cellular viability was assessed using adenosine 5'triphosphate content and protein synthesis in lung slices. Metabolites and remaining parent compounds in the tissue samples were analyzed by HPLC with UV detection.

Results: BDP was hydrolyzed to the pharmacologically active metabolite beclomethasone-17monopropionate (BMP) and, predominantly, to inactive beclomethasone (BOH). $\mathrm{CIC}$ was hydrolyzed initially to des-CIC with a slower rate compared to BDP. A distinctly smaller amount (approximately 10 -fold less) of fatty acid esters were formed by BMP (and/or BOH) than by BUD or des-CIC. The highest relative amounts of fatty acid esters were detected for BUD. For FP, no metabolites were detected at any time point. The amount of drug-related material in lung tissue (based on initial concentrations) at $24 \mathrm{~h}$ was highest for $\mathrm{ClC}$, followed by BUD and FP; the smallest amount was detected for BDP.

Conclusion: The in vitro metabolic pathways of the tested ICS in human lung tissue were differing. While FP was metabolically stable, the majority of BDP was converted to inactive polar metabolites. The formation of fatty acid conjugates was confirmed for BMP (and/or BOH), BUD, and des-CIC.

\section{Background}

Inhaled corticosteroids (ICS) are the standard first-line anti-inflammatory therapy for the management of persistent asthma [1]. ICS increase pulmonary function and decrease airway hyperresponsiveness, symptom severity, acute exacerbations [2], hospitalizations, and asthma mortality rates [3]. Binding of an ICS to the glucocorticoid receptor in the lung leads to a pleiotropic reduction in the expression of multiple pro-inflammatory pathways by reversing histone acetylation of the activated genes $[4,5]$. 
The preferred way to administer a corticosteroid in asthma therapy is by inhalation. This route delivers drug straight to the lung for local activity and minimizes the systemic side effects associated with oral administration. Nevertheless, even treatment with ICS may cause both local and systemic side effects. Deposition of the drug in the oropharynx may cause local side effects, including oropharyngeal candidiasis, dysphonia, reflex cough, bronchospasm, and pharyngitis [6,7]. Direct absorption of the active drug into the systemic circulation across the mucosa of the oropharynx and airways or absorption of swallowed drug via the gastrointestinal tract may result in systemic side effects. Skin thinning and bruising, cataracts, growth retardation, accelerated bone loss, and hypothalamic-pituitary-adrenal axis suppression have been observed in patients receiving long-term, high-dose ICS treatment $[8,9]$.

Pharmacokinetic (PK) properties of ICS are related to safety and efficacy of the drug. The efficacy of an ICS is dependent on high glucocorticoid receptor binding, high pulmonary deposition and retention, enhanced lipophilicity, and lipid conjugation. Safety is optimized by low oral bioavailability, high protein binding, and rapid systemic clearance $[10,11]$. Beclomethasone dipropionate (BDP), budesonide (BUD), and fluticasone propionate (FP) are widely used ICS and have been on the market in Asia, America, and Europe for several years. Ciclesonide (CIC) is a novel, nonhalogenated ICS that has been developed to incorporate PK properties that lead to high efficacy and an improved safety profile [11-15]. The prodrug CIC is administered as an inactive parent compound to the lower airways, where it is converted to its pharmacologically active metabolite desisobutyryl-ciclesonide (des-CIC) by endogenous esterases [12,16-19] Des-CIC has a high affinity to the glucocorticoid receptor, comparable with beclomethasone-17-monopropionate (BMP), whereas BUD has a slightly lower and FP a higher binding affinity [11]. Within the lung cells, des-CIC and BUD undergo reversible esterification with fatty acids at the C-21 position of the molecules. The formed fatty acid conjugates may serve as a depot that slowly releases desCIC and BUD in the lung [16,20-23] Hydrolysis of BDP at positions 17 and 21 results in three different metabolites, ie $\mathrm{BMP}$, beclomethasone-21-monopropionate (21-BMP), and beclomethasone ( $\mathrm{BOH})$. BMP is the active metabolite, whereas $\mathrm{BOH}$ and 21-BMP have a very low binding affinity to the glucocorticoid receptor $[11,24]$.

In the present study we investigated the in vitro metabolism of BDP, BUD, CIC, and FP in the target organ lung, using human lung precision-cut tissue slices, to evaluate the major factors influencing the retention time of these ICS in the lung.

\section{Methods \\ Materials}

BUD, CIC, des-CIC, des-CIC-palmitate, des-CIC-oleate, and FP were synthesized by ALTANA Pharma AG, Konstanz, Germany. BDP and BMP were obtained from MDS Pharmaservices (Fehraltorf, Switzerland).

Waymouth's MB752/1 powdered medium without phenol red was purchased from Gibco Laboratories (Grand Island, NY, USA). Fetal bovine serum and Fungi-Bact solution were purchased from Irvine Scientific (Santa Ana, CA, USA). Electrophoresis-grade agarose was obtained from ICN Biochemicals (Cleveland, OH, USA), and tracheal balloon catheters were obtained from Mallinchrodt Critical Care (Glen Falls, NY, USA). The dynamic roller culture incubators, Brendel/Vitron tissue slicer, titanium roller inserts, cordless coring tools, and V-7 cold preservation solution were manufactured by Vitron, Inc. (Tucson, AZ, USA). Water was Millipore filtered. All other reagents were obtained from Merck (Darmstadt, Germany) and were of professional analysis quality except methanol and ethanol, which were HPLC gradient grade.

\section{Human tissue collection}

The American Association of Human Tissue Users supplied three lungs. The lungs were accepted by The American Association of Human Tissue Users from American procurement agencies that followed donor consent procedures according to accepted medical and ethical standards as outlined by the Uniform Anatomical Gift Act. Tissues were negative for infectious agents including HIV and hepatitis. Organs were obtained under transplantation, but were not viable for transplantation because of anatomic irregularities, histological findings, age of donor, time in transit, or health status of the recipient.

\section{Preparation of tissue slices}

Lung slices were prepared at Vitron, Inc. (Tucson, AZ, USA) as previously described [25]. In brief, the lungs were inflated with 2 to $4 \mathrm{~L}$ of $0.5 \%$ agar/Waymouth's medium mixture at $37^{\circ} \mathrm{C}$ and were stored at $4{ }^{\circ} \mathrm{C}$ for 30 to $60 \mathrm{~min}$. Each lung was cut into $2 \mathrm{~cm}$ thick slabs, placed on dental wax, and tissue cylinders of $8 \mathrm{~mm}$ diameter were prepared using a cordless coring tool. $400 \mu \mathrm{m}$ thick slices were cut from the cylinders using a Brendel-Vitron tissue slicer [26].

\section{Tissue incubation}

Three lung slices from each donor were floated onto titanium roller inserts, blotted, and loaded horizontally into glass scintillation vials containing $1.7 \mathrm{~mL}$ of Waymouth's MB752/1 tissue culture medium fortified with 10\% fetal calf serum, $0.35 \mathrm{~g} / \mathrm{L}$ L-glutamine, $10 \mathrm{~mL} / \mathrm{L}$ Fungi-Bact, and $84 \mu \mathrm{g} / \mathrm{mL}$ gentamicin sulfate. 
Three vials were used per substrate and time point. A stock solution of the substrate was added prior to dispensing the media into vials. The final substrate concentration was $25 \mu \mathrm{M}$. The vials were closed with caps that had central 2 $\mathrm{mm}$ holes, placed in a Dynamic Organ Culture incubator (Vitron, Inc., Tucson, AZ, USA) at $37^{\circ} \mathrm{C}$, and exposed to $95 \% \mathrm{O}_{2}$ and $5 \% \mathrm{CO}_{2}$. The human tissue slices were incubated for 2, 6, and $24 \mathrm{~h}$, and cellular viability of human lung slices was assessed using separate incubation samples (see assessment of cellular viability). Slices and media from each vial were harvested separately into separate tubes and stored at $-80^{\circ} \mathrm{C}$. Metabolism samples containing either tissue or media were shipped on dry ice from Vitron, Inc. to ALTANA Pharma AG for analysis.

\section{Assessment of cellular viability}

Cellular viability was determined by measuring adenosine 5 '-triphosphate (ATP) content and protein synthesis in human lung tissue. ATP content was measured using a luciferin-luciferase assay [27]. Tissue slices were homogenized in $1 \mathrm{~mL} \mathrm{10 \%} \mathrm{trichloroacetic} \mathrm{acid} \mathrm{and} \mathrm{were} \mathrm{immedi-}$ ately frozen at $-80^{\circ} \mathrm{C}$ until ATP content was measured using a luminometer. Protein synthesis was measured by the addition of $0.3 \mu \mathrm{Ci}\left[{ }^{3} \mathrm{H}\right]$-leucine/mL to tissue slice incubations. Tissue slices were sonicated in $1 \mathrm{~mL}$ distilled water, and a $20 \mu \mathrm{L}$ aliquot was used to determine protein content. The remaining homogenate was precipitated with $5 \%(\mathrm{w} / \mathrm{v})$ perchloric acid. Samples were centrifuged and the resultant pellet was washed 3 times. The final pellet was dissolved in $0.5 \mathrm{~mL}$ of $0.5 \mathrm{~N}$ sodium hydroxide and neutralized with $125 \mu \mathrm{L}$ of $2 \mathrm{~N}$ hydrochloric acid. Incorporation of the labeled amino acid into acid-precipitable material was measured by counting a $0.5 \mathrm{~mL}$ aliquot of the sample in Bio-Safe II scintillation cocktail. Viability of lung tissue slices was expressed per milligram of protein.

\section{Sample preparation}

The volume of medium was measured at the end of the incubation period. Samples of the incubation medium were prepared by mixing $60 \mu \mathrm{L}$ of medium with $60 \mu \mathrm{L}$ of solution for precipitation $\left(0.2 \mathrm{M} \mathrm{ZnSO}_{4} / \mathrm{ACN}, 1: 1\right)$. Following centrifugation for $5 \mathrm{~min}(1,000 \times \mathrm{g}$ Model5414, Eppendorf) at room temperature, the supernatant was assayed by high performance liquid chromatography (HPLC). Tissue samples were homogenized in $1 \mathrm{~mL}$ of methanol using ultrasonic treatment (Branson Sonifier 250; Branson Ultrasonics Corporation, Danbury, Conn) and were centrifuged for $5 \mathrm{~min}(1,000 \times \mathrm{g}$ Model5414, Eppendorf) at room temperature. After centrifugation, $333 \mu \mathrm{L}$ of the supernatant was dried under a gentle stream of nitrogen at $45^{\circ} \mathrm{C}$ and resuspended in $75 \mu \mathrm{L}$ of methanol before analysis.

\section{High-performance liquid chromatography analysis}

HPLC analysis was performed using a Hewlett-Packard HP1090 liquid chromatograph (Agilent Technologies Deutschland GmbH, Waldbronn, Germany) with an ultraviolet (UV) detector monitoring at $242 \mathrm{~nm}$. Separation was achieved using a Hypersil MOS column (125 × 4 $\mathrm{mm}, 5 \mu \mathrm{m}$ ) (Thermo Electron Corp., Dreieich, Germany). A water/ethanol gradient system was run from 30 to $90 \%$ ethanol in $10 \mathrm{~min}$ followed by an isocratic phase (6 min) with $90 \%$ ethanol at a flow rate of $0.75 \mathrm{~mL} / \mathrm{min}$. $25 \mu \mathrm{L}$ of the tissue extract or $100 \mu \mathrm{L}$ of the supernatant of the medium were injected onto the column. This system was suitable to assay all parent compounds used as substrate and the fatty acid conjugated metabolites. BOH, BDP, $\mathrm{BMP}, \mathrm{BUD}, \mathrm{CIC}$, des-CIC, des-CIC-oleate, and FP were used as reference compounds. All these compounds have a similar UV response at $242 \mathrm{~nm}$.

\section{Results \\ Donor information}

Human lung tissues from 3 human donors were used to study metabolism of four ICS. Donor profiles are summarized in Table 1.

\section{Cellular viability}

Viability of tissue samples was assessed using ATP content and protein synthesis levels in human lung after incubation of tissue samples with ICS for 2, 6, and $24 \mathrm{~h}$. ATP content ranged from 17.0 to $40.7 \mathrm{nmol} / \mathrm{mg}$ protein. Protein synthesis displayed a linear mean increase from 1,930 $\mathrm{dpm} / \mathrm{mg}$ protein after $2 \mathrm{~h}$ of incubation to $15,409 \mathrm{dpm} /$ mg protein after $24 \mathrm{~h}$ of incubation (Table 2). Fisher and colleagues have reported that ATP content should exceed $10 \mathrm{nmol} / \mathrm{mg}$ protein and protein synthesis should be linear over time in viable human lung tissue [26]. Therefore, all lung tissue samples used in this study were highly viable.

\section{Analytics}

The HPLC system was suitable to assay all parent compounds used as substrate as well as all known major metabolites. However, the inactive 21-BMP was not available as reference compound. It was assumed that the retention time of BMP and 21-BMP is similar and in some cases shoulders in the BMP peaks were observed. Assum-

Table I: Donor characteristics

\begin{tabular}{|c|c|c|c|}
\hline Donor & $\mathbf{I}$ & 2 & 3 \\
\hline Age & 19 & 48 & 43 \\
\hline Sex & Male & Male & Female \\
\hline Race & Hispanic & Caucasian & Caucasian \\
\hline Cause of death & Cerebrovascular & Cerebrovascular & Cerebrovascular \\
\hline Medical history & Healthy & Healthy & Healthy \\
\hline Medications & None & None & None \\
\hline
\end{tabular}


Table 2: Viability parameters

\begin{tabular}{cccc}
\hline $\begin{array}{c}\text { Incubation } \\
\text { time [h] }\end{array}$ & $\begin{array}{c}\text { Lung } \\
\text { from } \\
\text { donor }\end{array}$ & $\begin{array}{c}\text { ATP Content } \\
\text { [nmoles ATP/ } \\
\text { mg protein] }\end{array}$ & $\begin{array}{c}\text { Protein } \\
\text { Synthesis [dpm/ } \\
\text { mg protein] }\end{array}$ \\
\hline \multirow{2}{*}{2} & 1 & $36.3-37.4$ & $2,688-2,797$ \\
& 2 & $36.5-40.2$ & $1,924-2,355$ \\
$908-939$
\end{tabular}

Data from $n=3$ tissue slices per lung.

ing coeluation of BMP and 21-BMP from the HPLC column and assuming a very similar UV response, the BMP peak may represent partly 21-BMP. Therefore, the amount of active BMP may be overestimated.

Furthermore, the lipophilic fatty acid conjugated metabolites as well as the polar $\mathrm{BOH}$ were detectable. Matrix peaks did not influence the detection of drug-related peaks. Results of control samples confirmed the stability of the tested compounds during incubation under the used conditions. Furthermore, matrix peaks of tissue slices and medium could be identified using the HPLC system. Interday variability of all analytes was less than $15 \%$.

\section{Drug-related material in the incubation medium}

Substrates were analyzed in medium after an incubation time of 2, 6, and $24 \mathrm{~h}$. BUD and FP were stable, ie no metabolites were detected in medium, whereas the relative amounts of CIC and BDP decreased over time and metabolites were detected in the incubation medium (Table 3). CIC was metabolized to des-CIC, which was the only metabolite of CIC in the medium. At the end of the incubation period $(24 \mathrm{~h})$, the parent compound accounted for $37 \%$ of CIC-related material in the medium. In contrast, at $24 \mathrm{~h}$ of incubation, there was no BDP detectable in the medium, whereas its metabolites accounted for $91.6 \%(\mathrm{BOH})$ and $8.4 \%$ (BMP) of BDPrelated material (Table 3 ).

\section{Drug-related material in tissue samples}

In contrast to the medium samples, fatty acid esters were detectable in tissue extracts. Furthermore, metabolic differences between the ICS were obvious in tissue extracts. The relative amounts of BDP, BUD, CIC, FP, and their respective metabolites in human lung tissue at 2, 6, and $24 \mathrm{~h}$ of incubation are presented in Table 4. Absolute
Table 3: Relative drug amounts [\% of total UV areas] in medium

\begin{tabular}{lcccc}
\hline & $\begin{array}{c}\text { Incubatio } \\
\text { n time [h] }\end{array}$ & $\begin{array}{c}\text { Parent } \\
\text { compound }\end{array}$ & $\begin{array}{c}\text { Active } \\
\text { compound }\end{array}$ & $\begin{array}{c}\text { Inactive } \\
\text { metabolite }\end{array}$ \\
\hline Beclomethasone & 2 & $60.9 \pm 7.0$ & $35.7 \pm 6.4$ & $3.4 \pm 0.8$ \\
Dipropionatel & 6 & $19.1 \pm 8.3$ & $53.5 \pm 4.3$ & $27.3 \pm 4.7$ \\
& 24 & - & $8.4 \pm 5.3$ & $91.6 \pm 5.3$ \\
& 2 & $94.9 \pm 1.3$ & $5.1 \pm 1.3$ & - \\
\hline Ciclesonide & 6 & $83.2 \pm 5.5$ & $16.8 \pm 5.5$ & - \\
& 24 & $37.4 \pm 7.8$ & $62.6 \pm 7.8$ & - \\
& & & & \\
\hline
\end{tabular}

Data are presented as mean \pm standard deviation $(n=9)$.

No metabolites of Bud and FP were detected in medium.

IThe active compound of BDP may be overestimated as inactive 21-BMP may be included.

amounts of the drugs and their metabolites are presented in Figure 1 and Table 5.

BDP was hydrolyzed to active BMP and to pharmacologically inactive $\mathrm{BOH}$ and 21-BMP. As mentioned above, 21BMP could not be quantified due to coeluation in the BMP peak and therefore the amount of active BMP may be overestimated. BMP was the major metabolite at $2 \mathrm{~h}$ and $6 \mathrm{~h}$, whereas $\mathrm{BOH}$ was the major metabolite at $24 \mathrm{~h}$. At 24 h, BMP accounted for $26.6 \%$ and BOH for $64.9 \%$ of BDPrelated material. Fatty acid esters were formed by BMP (and/or $\mathrm{BOH}$ ), but the amount was distinctly smaller (approximately 10-fold less) when compared with the amount of fatty acid esters found for BUD and des-CIC. The relative amount of BUD decreased over time and similar amounts of BUD and its fatty acid esters were detected in the tissue at $24 \mathrm{~h}$. CIC was initially hydrolyzed to desCIC, which subsequently was conjugated with fatty acids. Both the relative and the mean amounts of des-CIC and fatty acid conjugates of des-CIC increased over time. For $\mathrm{FP}$, no metabolites were detected in the tissue extract. FP was metabolically stable in this test system. Furthermore, the intracellular amount of FP was similar at all time points during incubation.

Based on these results, active metabolites were formed by $\mathrm{BDP}$ and CIC. The enzymatic cleavage of BDP to BMP was faster than the formation of des-CIC from CIC (see $2 \mathrm{~h}$ data in Table 4). The active metabolite BMP was further metabolized to $\mathrm{BOH}$, whereas the active metabolite des$\mathrm{CIC}$ and the active compound BUD formed intracellular fatty acid esters. These esters may serve as precursors of the active compounds because the conjugation is a reversible process. Parent compounds, active metabolites, and fatty acid esters form a pool of active drug. At $24 \mathrm{~h}$, the mean absolute values for the active drug pools in the lung tissue slices were $9.31 \mathrm{nmol}$ for BDP, $17.95 \mathrm{nmol}$ for BUD, $24.43 \mathrm{nmol}$ for CIC, and $9.51 \mathrm{nmol}$ for FP. The pool of active drug (including parent compound, active metabolite, and fatty acid esters) was larger for CIC than for the other ICS. 
Table 4: Relative drug amounts [\% of total UV areas] in human lung tissue slices

\begin{tabular}{|c|c|c|c|c|c|}
\hline & Incubation time [h] & Parent compound & Active compound & Fatty acid esters & Inactive metabolite \\
\hline Beclomethasone & 2 & $21.4 \pm 9.8$ & $72.1 \pm 9.2$ & $2.9 \pm 1.1$ & $3.6 \pm 0.8$ \\
\hline \multirow[t]{2}{*}{ dipropionate $^{2}$} & 6 & $9.2 \pm 7.9$ & $66.6 \pm 17.7$ & $4.4 \pm 2.6$ & $19.8 \pm 10.0$ \\
\hline & 24 & - & $26.6 \pm 16.8$ & $8.5 \pm 7.8$ & $64.9 \pm 14.4$ \\
\hline \multirow[t]{3}{*}{ Budesonidel } & 2 & \multicolumn{2}{|c|}{$72.2 \pm 10.8$} & $27.8 \pm 10.8$ & - \\
\hline & 6 & \multicolumn{2}{|c|}{$64.9 \pm 12.0$} & $35.1 \pm 12.0$ & - \\
\hline & 24 & \multicolumn{2}{|c|}{$53.6 \pm 11.5$} & $46.4 \pm 11.5$ & - \\
\hline \multirow[t]{3}{*}{ Ciclesonide } & 2 & $85.0 \pm 3.4$ & $9.9 \pm 1.4$ & $5.1 \pm 2.1$ & - \\
\hline & 6 & $73.5 \pm 4.7$ & $17.9 \pm 1.5$ & $8.6 \pm 4.1$ & - \\
\hline & 24 & $40.0 \pm 7.6$ & $31.5 \pm 2.4$ & $28.5 \pm 8.8$ & - \\
\hline Fluticasonel & 2 & \multicolumn{2}{|c|}{$100 \pm 0.0$} & - & - \\
\hline \multirow[t]{2}{*}{ propionate } & 6 & \multicolumn{2}{|c|}{$100 \pm 0.0$} & - & - \\
\hline & 24 & \multicolumn{2}{|c|}{$100 \pm 0.0$} & - & - \\
\hline
\end{tabular}

Data are presented as mean \pm standard deviation $(n=9)$.

IParent and active compound.

2The active compound of BDP may be overestimated as inactive 2I-BMP may be included.

\section{Discussion}

Several pharmacokinetic and pharmacodynamic parameters, including bioavailability, receptor binding-affinity, and elimination half-life contribute to the safety and efficacy profile of an ICS. While developing topical corticosteroids for the treatment of asthma, a delicate balance between safety and efficacy must be considered. In the present study, differences in the in vitro metabolism of currently available ICS in human lung tissue slices were evaluated with respect to their local effect on pulmonary residence time.
Results of the present study confirm the hypothesis that the metabolism of BDP, BUD, CIC, and FP in the lung is considerably different. This was shown in a direct comparison using human precision-cut tissue slices. Unlike BUD and FP, which are active in their parent form, both BDP and CIC are activated in the airways by esterases to pharmacologically active BMP or des-CIC, respectively. Results from this study confirm previous findings showing that $\mathrm{BDP}$ is hydrolyzed to $\mathrm{BOH}$ in the cytosol of human lung cells [24]. However, in the current study the formation of $\mathrm{BOH}$ was much faster, as was also the case in a previous

Table 5: Total amount [nmol/vial] of drug related compounds in human lung tissue slices

\begin{tabular}{|c|c|c|c|c|c|}
\hline & Incubation time [h] & Parent compound & Active compound & Fatty acid esters & Inactive metabolite \\
\hline Beclomethasone & 2 & $2.19 \pm 0.83$ & $7.88 \pm 2.21$ & $0.90 \pm 0.46$ & $0.38 \pm 0.06$ \\
\hline \multirow[t]{2}{*}{ dipropionate $^{2}$} & 6 & $0.72 \pm 0.46$ & $7.78 \pm 3.80$ & $0.50 \pm 0.40$ & $1.89 \pm 0.49$ \\
\hline & 24 & - & $2.73 \pm 2.22$ & $0.87 \pm 0.96$ & $5.72 \pm 1.20$ \\
\hline \multirow[t]{3}{*}{ Budesonidel } & 2 & \multicolumn{2}{|c|}{$7.32 \pm 0.99$} & $3.17 \pm 2.00$ & - \\
\hline & 6 & \multicolumn{2}{|c|}{$8.41 \pm 2.15$} & $5.39 \pm 4.03$ & - \\
\hline & 24 & \multicolumn{2}{|c|}{$9.04 \pm 1.14$} & $8.91 \pm 5.15$ & - \\
\hline \multirow[t]{3}{*}{ Ciclesonide } & 2 & $6.46 \pm 0.92$ & $0.76 \pm 0.2$ & $0.40 \pm 0.20$ & - \\
\hline & 6 & $8.86 \pm 1.68$ & $2.17 \pm 0.51$ & $1.10 \pm 0.73$ & - \\
\hline & 24 & $9.53 \pm 1.70$ & $7.61 \pm 1.31$ & $7.29 \pm 3.79$ & - \\
\hline Fluticasonel & 2 & \multicolumn{2}{|c|}{$8.42 \pm 1.26$} & - & - \\
\hline \multirow[t]{2}{*}{ propionate } & 6 & \multicolumn{2}{|c|}{$8.62 \pm 2.20$} & - & - \\
\hline & 24 & \multicolumn{2}{|c|}{$9.51 \pm 2.22$} & - & - \\
\hline
\end{tabular}

Data are presented as mean \pm standard deviation $(n=9)$.

IParent and active compound.

${ }^{2}$ The active compound of BDP may be overestimated as inactive 2I-BMP may be included. 

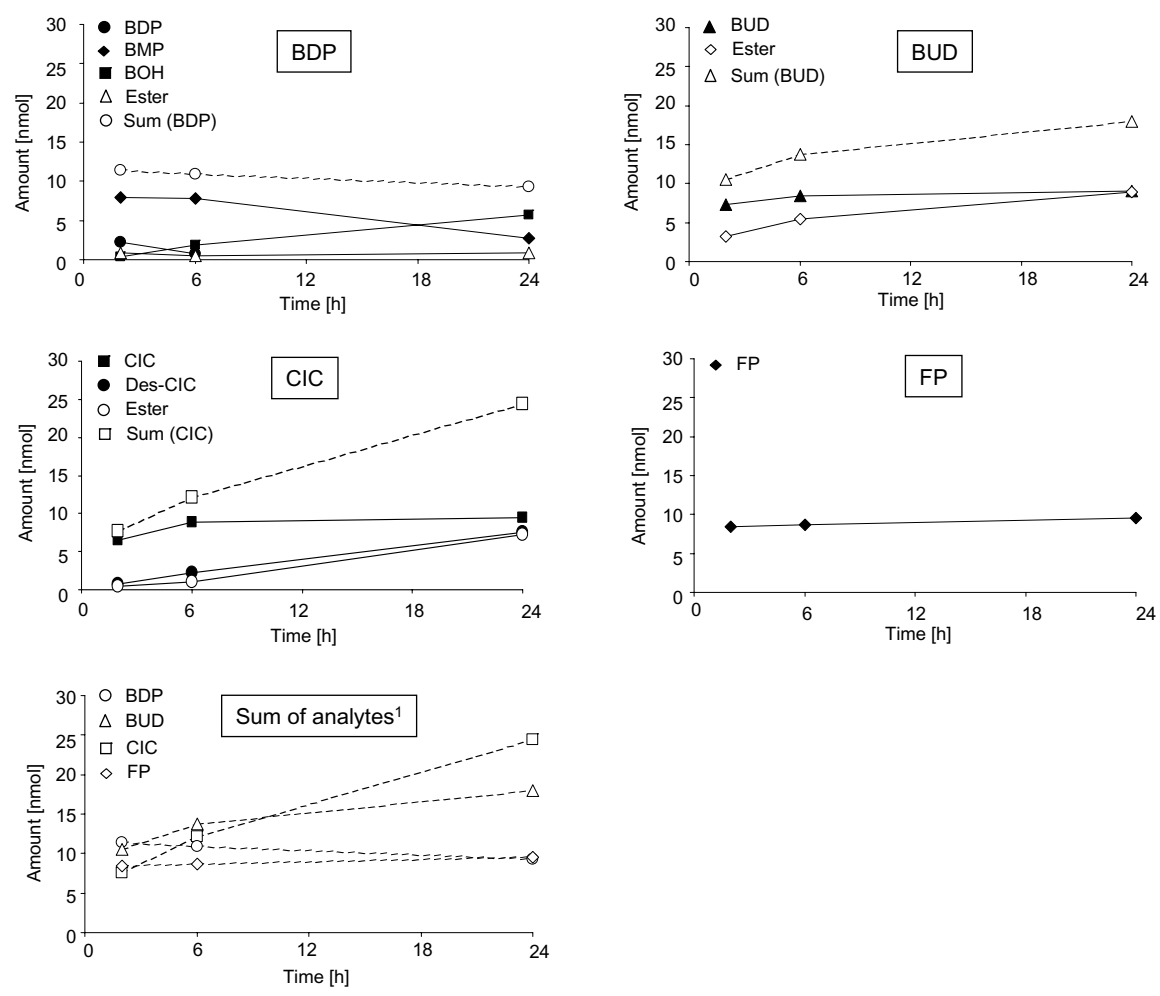

Figure I

Mean amounts of parent compounds and metabolites in lung tissue slices over time. Initial substrate concentration: $25 \mu \mathrm{M}$. 'Sum of analytes = Pool for active drug detectable in human lung slices after 2, 6 , and $24 \mathrm{~h}$ of incubation. BDP = beclomethasone dipropionate, $\mathrm{BUD}=$ budesonide, $\mathrm{CIC}=$ ciclesonide, $\mathrm{FP}=$ fluticasone propionate.

study in rat lung precision-cut tissue slices [20]. The majority of BMP was inactivated to BOH and only a small amount of esters was formed. In contrast, a considerable amount of des-CIC fatty acid conjugates were detected. Experimentally it was not clear whether BMP, 21-BMP and/or $\mathrm{BOH}$ was conjugated with fatty acids, because the amounts of these metabolites were too low for further characterization by mass spectrometry. Similar to desCIC, BUD formed intracellular free fatty acid esters. A prerequisite for the formation of fatty acid esters is the presence of a steric hindrance-free hydroxyl group at the C-21 position of the ICS. BMP, BOH, BUD, and des-CIC have a free C-21 hydroxyl group that can be reversibly esterified. BUD is the only parent compound with a free C-21 hydroxyl group and, therefore, esterification can start directly once BUD has been taken up by the cell. This may explain the high portion of fatty acid esters of BUD at early time points. FP, which has no free C-21 hydroxyl group, was metabolically stable in the test system because no metabolites were detectable in the tissue and incubation medium.
In this study, a relatively high initial concentration of ICS was used to investigate metabolism in the lung. The concentration of $25 \mu \mathrm{M}$ was the same as used in previous studies investigating CIC metabolism in precision-cut tissue slices of human and rat lungs $[16,20]$. In these studies, the use of ${ }^{14} \mathrm{C}$-ciclesonide allowed the estimation of recovery and mass balance. In the present study, although incubations were carried out with non-radiolabelled CIC, the same methods and conditions were used. The overall metabolism rate of $\mathrm{CIC}$ in the current investigation was lower than in the previous study, whereas the viability of the cells was comparable in both studies [16]. At the end of the incubation period, the relative percentage of desCIC was very similar in both studies (29\% vs $32 \%$ ).

The concentration of corticosteroids in the systemic circulation after inhalation depends on the pharmacokinetic properties of the drug, the formulation, and the device, but is in general much lower than the concentration of ICS used in the current study $(25 \mu \mathrm{M})$. For example, in healthy volunteers, inhalation of CIC $1280 \mu \mathrm{g}$ via 
metered-dose inhaler (MDI) resulted in a $\mathrm{C}_{\max }$ of $5.07 \mu \mathrm{g} /$ $\mathrm{L}$ (9.4 nmol/L, geometric mean) [28] and inhalation of FP $1000 \mu \mathrm{g}$ via Diskhaler ${ }^{\circledR}$ resulted in a $\mathrm{C}_{\text {max }}$ of $0.38 \mu \mathrm{g} / \mathrm{L}$ (0.76 nmol/L, mean) [29]. However, low serum concentrations of corticosteroids do not necessarily mimic the situation in the lung. After inhalation, concentrations of ICS may be significantly higher in the lung compared with concentrations in serum [6]. This was confirmed in three different studies investigating the concentration of drugs in resected lung tissue samples as well as in serum following single inhalation of ICS. After inhalation of $1600 \mu \mathrm{g}$ BUD via Nebuhaler ${ }^{\circledR}$, the mean concentration of BUD in lung tissue obtained from resections was $5.5 \mathrm{nmol} / \mathrm{kg}$ compared with $0.63 \mathrm{nmol} / \mathrm{kg}$ in blood plasma [30]. In a similar study, FP concentrations of more than $20 \mathrm{ng} / \mathrm{g}$ central lung tissue were detected in a few patients, while the mean concentration in serum at that time was approximately $0.02 \mathrm{ng} / \mathrm{mL}$ [31]. After inhalation of $1 \mathrm{mg}$ BDP via a MDI (Aerobec ${ }^{\circledR}$ ), the median concentration of BMP in bronchial tissue and serum was $4.4 \mathrm{ng} / \mathrm{g}$ tissue and $1.2 \mathrm{ng} /$ $\mathrm{mL}$, respectively [33]. Although quantitative differences cannot be excluded, the metabolic pathways of the tested ICS in the lung should be unaffected by the chosen initial drug concentration.

Lipophilicity and lipid conjugation are two distinct PK parameters that can affect the absorption rate of an ICS across pulmonary membranes and influence retention time in the lung. Slow absorption from the lung or pulmonary retention of the drug by some mechanism may enhance the anti-inflammatory effects of the drug without the unwanted systemic side effects.

The amount of FP detected in the lung tissue remained constant during the investigated period of 2 to $24 \mathrm{~h}$. The relative distribution of FP between the tissue and the incubation medium may depend on the lipophilicity and the glucocorticoid receptor affinity of the compound. Because the concentration of the drug is considered to be high compared with the number of available receptor sites, the main factor for uptake into the cells and intracellular residence seems to be the lipophilicity of the drug $(\log \mathrm{D}$ of FP at pH 7.4 is 3.7 [20]). However, in the present study no data for the early distribution phase were generated that would allow a correlation between lipophilicity and distribution. The uptake of FP and CIC into human alveolar type II epithelial cells (A549) was investigated separately [32]. In contrast to FP, all other tested drugs were metabolized in the lung tissue. The active metabolite of BDP, $\mathrm{BMP}$, was further converted to fatty acid esters and mainly to inactive polar metabolites. Over time, the concentration of drug-related compounds decreased in the tissue and the formed polar metabolites diffused into the medium (91.6\% BOH and 8.4\% BMP by $24 \mathrm{~h})$.
The intracellular fatty acid conjugation of BUD and desCIC dramatically increased the lipophilicity of the drugs. No fatty esters were detectable outside the cells. Des-CIColeate $(\log \mathrm{D} 13.0)$ was about 5 -fold more lipophilic than BUD-oleate $(\log D$ 12.3) [20]. The absolute drug amounts in the lung slices at the end of the incubation period may be correlated with their lipophilicity. CIC $(\log D$ 6.1) and des-CIC fatty acid esters are highly lipophilic [20], which suggests that these compounds would be retained in the lung for a prolonged period of time. Furthermore, both compounds are precursors of des-CIC and may thus serve as a pool of active drug. Although the amount of active compounds measured in the human lung tissue slices were similar for BUD, des-CIC, and FP, the amount of drug-related material detected in the tissue after $24 \mathrm{~h}$ of incubation was higher for CIC than for the other tested ICS.

Results from this study confirm previous findings that both CIC and BUD reversibly form conjugates with fatty acids $[16,19,21-23]$

Unlike BDP and CIC, both BUD and FP are inhaled as active compounds. Therefore, drug exposure in the oropharyngeal region may be much higher for BUD and FP than for the active metabolites of BDP [33] and CIC $[34,35]$. The activation of BDP in the oropharynx seems to be fast, because similar amounts of BDP and BMP were detected in mouth rinsing solutions directly after inhalation of BDP $1000 \mu \mathrm{g}$ via MDI [33]. In contrast, the activation of CIC in the oropharynx is very low. Only traces of des-CIC were detected in mouth rinsing solutions directly after inhalation of CIC $800 \mu \mathrm{g}$ via MDI [34,35]. Higher concentrations of BMP compared to des-CIC in the oropharynx might be explained by differences in the velocity of BMP and des-CIC formation as observed in the present study. Administration of CIC as an inactive parent compound and the low oropharyngeal deposition of desCIC may explain the low incidence of oropharyngeal side effects observed in clinical studies [36].

\section{Conclusion}

In summary, when developing ICS it is necessary to consider the overall balance between safety and efficacy. In this study we saw clear differences between the investigated ICS regarding the residence time of the drugs within the lung tissue slices, because of their different metabolism, lipophilicity, and ability to form lipid conjugates. Highly lipophilic drugs will likely contribute to a high rate of absorption within the lung. Once in the lung, reversible fatty acid conjugation may prolong the local anti-inflammatory activity by slowly releasing active drug from the fatty acid ester depot. Of the currently available ICS, only $\mathrm{CIC}$ and BUD undergo lipid conjugation. This concept 
supports the once-daily dosing efficacy of these drugs that was observed in clinical studies $[37,38]$.

\section{Abbreviations}

ATP-Adenosine triphosphate.

BDP- Beclomethasone dipropionate.

BMP-Beclomethasone-17-monopropinate.

BOH- Beclomethasone.

BUD- Budesonide.

CIC- Ciclesonide.

FP- Fluticasone propionate.

HPLC- High performance liquid chromatography.

ICS- Inhaled corticosteroid.

UV- Ultra violet.

\section{Competing interests}

RN and NM are employees of Nycomed GmbH (formerly ALTANA Pharma AG), Konstanz, Germany, who sponsored this study. The author RF has received honoraria from ALTANA Pharma AG for conducting part of this study.

\section{Authors' contributions}

RN planned the experiments, carried out the HPLC and data analyses, and drafted the manuscript. RF prepared the lung tissue slices, carried out the tissue incubations and the cell viability tests. NM, as head of department, was involved in developing experimental plans and manuscript revisions. All authors read and approved the final manuscript.

\section{Acknowledgements}

The authors thank Dr Tanja Henrichs and Dr Kathy B. Thomas (Nycomed $\mathrm{GmbH}$, Department Medical Writing, Konstanz, Germany) for helpful suggestions during the preparation of this article.

\section{References}

I. Williams SG, Schmidt DK, Redd SC, Storms W: Key clinical activities for quality asthma care. 2007 [http://www.cdc.gov/mmwr/ preview/mmwrhtml/rr5206al.htm].

2. Georgitis JW: The $\mathbf{1 9 9 7}$ Asthma Management Guidelines and therapeutic issues relating to the treatment of asthma. Chest 1999, II 5:210-217.

3. Suissa S, Ernst P: Inhaled corticosteroids: impact on asthma morbidity and mortality. J Allergy Clin Immunol 200I, 107:937-944.

4. Umland SP, Schleimer RP, Johnston SL: Review of the molecular and cellular mechanisms of action of glucocorticoids for use in asthma. Pulm Pharmacol Ther 2002, 15:35-50.
5. Pelaia G, Vatrella A, Cuda G, Maselli R, Marsico SA: Molecular mechanisms of corticosteroid actions in chronic inflammatory airway diseases. Life Sci 2003, 72:1549-156I.

6. Derendorf H: Pharmacokinetic and pharmacodynamic properties of inhaled corticosteroids in relation to efficacy and safety. Respir Med 1997, 91:22-28.

7. Barnes PJ, Pedersen S, Busse WW: Efficacy and safety of inhaled corticosteroids. New developments. Am J Respir Crit Care Med 1998, 157:SI-S53.

8. Lipworth BJ: Systemic adverse effects of inhaled corticosteroid therapy: a systematic review and meta-analysis. Arch Intern Med 1999, 159:941-955.

9. Newman SP: Deposition and effects of inhaled corticosteroids. Clin Pharmacokinet 2003, 42:529-544.

10. Rohatagi S, Appajosyula S, Derendorf H, Szefler S, Nave R, Zech K, Banerji D: Risk-benefit value of inhaled glucocorticoids: a pharmacokinetic/pharmacodynamic perspective. J Clin Pharmacol 2004, 44:37-47.

II. Derendorf H, Nave R, Drollmann A, Cerasoli F, Wurst W: Relevance of pharmacokinetics and pharmacodynamics of inhaled corticosteroids to asthma. Eur Respir J 2006, 28: $1042-1050$.

12. Dent G: Ciclesonide (Byk Gulden). Curr Opin Investig Drugs 2002, 3:78-83.

13. Postma DS, Sevette C, Martinat Y, Schloesser N, Aumann J, Kafé H: Treatment of asthma by the inhaled corticosteroid ciclesonide given either in the morning or evening. Eur Respir J 200I, 17:1083-1088.

14. Kanniess F, Richter K, Böhme S, Jörres RA, Magnussen H: Effect of inhaled ciclesonide on airway responsiveness to inhaled AMP, the composition of induced sputum and exhaled nitric oxide in patients with mild asthma. Pulm Pharmacol Ther 200I, I4: I4I-I 47.

15. Larsen BB, Nielsen LP, Engelstätter R, Steinijans V, Dahl R: Effect of ciclesonide on allergen challenge in subjects with bronchial asthma. Allergy 2003, 58:207-2I2.

16. Nave R, Fisher R, Zech K: In Vitro metabolism of ciclesonide in human lung and liver precision-cut tissue slices. Biopharm Drug Dispos 2006, 27:197-207.

17. Mutch E, Nave R, Zech K, Williams FM: Esterases involved in the hydrolysis of ciclesonide in human tissues. Eur Respir J 2003, 22:267s-268s.

18. K D, R E, A. K: Ciclesonide: An on-site-activated steroid. In New drugs for asthma, allergy and COPD Edited by: Hansel TT and Barnes PJ. Basel, Karger; 2001:91-93.

19. Sato H, Nave R, Nonaka T, Mochizuki T, Takahama S, Kondo S: In vitro metabolism of ciclesonide in human nasal epithelial cells. Biopharm Drug Dispos 2007, 28:43-50.

20. Nave R, Hummel RP, Wohlsen A, Herzog R, Zech K: The active metabolite of ciclesonide, des-isobutyryl ciclesonide, forms highly lipophilic fatty acid conjugates in precision-cut rat lung slices. Am J Respir Crit Care Med 2004, 169:A91.

21. Nave R, Meyer W, Fuhst R, Zech K: Formation of fatty acid conjugates of ciclesonide active metabolite in the rat lung after 4-week inhalation of ciclesonide. Pulm Pharmacol Ther 2005, 18:390-396.

22. Miller-Larsson A, Mattsson H, Hjertberg E, Dahlbäck M, Tunek A, Brattsand R: Reversible fatty acid conjugation of budesonide. Novel mechanism for prolonged retention of topically applied steroid in airway tissue. Drug Metab Dispos 1998, 26:623-630.

23. Tunek A, Sjödin K, Hallström G: Reversible formation of fatty acid esters of budesonide, an antiasthma glucocorticoid, in human lung and liver microsomes. Drug Metab Dispos 1997, 25: $1311-1317$.

24. Würthwein G, Rohdewald P: Activation of beclomethasone dipropionate by hydrolysis to beclomethasone-17-monopropionate. Biopharm Drug Dispos 1990, I I:38I-394.

25. Fisher RL, Gandolfi AJ, Brendel K: Human liver quality is a dominant factor in the outcome of in vitro studies. Cell Biol Toxicol 2001, 17:179-189.

26. Fisher RL, Smith MS, Hasal SJ, Hasal KS, Gandolfi AJ, Brendel K: The use of human lung slices in toxicology. Hum Exp Toxicol 1994, 13:466-47|.

27. Kricka L): Clinical and biochemical applications of luciferases and luciferins. Anal Biochem 1988, 175:14-21. 
28. Nave R, Gunawardena KA, Zech K, Bethke TD: Pharmacokinetic disposition of inhaled ciclesonide and its metabolite desisobutyryl-ciclesonide in healthy subjects and patients with asthma are similar. Int / Clin Pharmacol Ther 2006, 44: I-7.

29. Mackie AE, McDowall JE, Falcoz C, Ventresca P, Bye A, Daley-Yates PT: Pharmacokinetics of Fluticasone Propionate inhaled via the Diskhaler ${ }^{\circledR}$ and Diskus $®$ Powder Devices in Healthy Volunteers. Clin Pharmacokinet 2000, 39:23-30.

30. Van Den Bosch JMM, Westermann CJ], Aumann J, Edsbäcker S, Tönnesson M, Selroos O: Relationship between lung tissue and blood plasma concentrations of inhaled budesonide. Biopharmaceutics and Drug Disposition 1993, 14:455-459.

31. Esmailpour N, Högger P, Rabe KF, Heitmann U, Nakashima M, Rohdewald $P$ : Distribution of inhaled fluticasone propionate between human lung tissue and serum in vivo. Eur Respir 1997, I0:1496-1499.

32. Nonaka T, Nave R, McCracken N, Kawashimo A, Katsuura Y: Ciclesonide uptake and metabolism in human alveolar type II epithelial cells (A549). BMC Pharmacol 2007, 7(I): I 2.

33. Holz $O$, Zühlke I, Einhaus M, Welker L, Kanniess F, Branscheid D, Nakashima M, Harrison LI, Jörres RA, Richter K, Magnussen H: Direct measurement of BDP and I7-BMP in bronchial and peripheral lung tissue after inhalation of HFA- vs CFC-driven aerosols. Pulmonary Pharmacology and Therapeutics 2004, | 7:233-238

34. Nave R, Zech K, Bethke TD: Lower oropharyngeal deposition of inhaled ciclesonide via hydrofluoroalkane metered-dose inhaler compared with budesonide via chlorofluorocarbon metered-dose inhaler in healthy subjects. Eur J Clin Pharmacol 2005, 61 :203-208.

35. Richter K, Kanniess F, Biberger C, Nave R, Magnussen H: Comparison of the oropharyngeal deposition of inhaled ciclesonide and fluticasone propionate in patients with asthma. J Clin Pharmacol 2005, 45: | 46- I52.

36. Engelstätter R, Escher A, Haefner D: Low incidence of oropharyngeal adverse events in asthma patients treated with ciclesonide. Eur Respir J 2005, 26:255s.

37. Chapman KR, Patel P, D'Urzo AD, Alexander M, Mehra S, Oedekoven C, Engelstätter R, Boulet LP: Maintenance of asthma control by once-daily inhaled ciclesonide in adults with persistent asthma. Allergy 2005, 60:330-337.

38. Mintz S, Alexander M, Li JH, Mayer PV: Once-daily administration of budesonide Turbuhaler was as effective as twice-daily treatment in patients with mild to moderate persistent asthma. J Asthma 2002, 39:203-210.

\section{Publish with Bio Med Central and every scientist can read your work free of charge}

"BioMed Central will be the most significant development for disseminating the results of biomedical research in our lifetime. "

Sir Paul Nurse, Cancer Research UK

Your research papers will be:

- available free of charge to the entire biomedical community

- peer reviewed and published immediately upon acceptance

- cited in PubMed and archived on PubMed Central

- yours - you keep the copyright 